Revista Eureka sobre Enseñanza y Divulgación de las Ciencias

ISSN: 1697-011X

revista.eureka@uca.es

Universidad de Cádiz

España

\title{
Diseño y evaluación de una intervención educativa sobre la pandemia de la COVID-19 y las medidas de prevención
}

Portillo-Blanco, Ane; Díez, José Ramón; Barrutia, Oihana; Garmendia, Mikel; Guisasola, Jenaro Diseño y evaluación de una intervención educativa sobre la pandemia de la COVID-19 y las medidas de prevención

Revista Eureka sobre Enseñanza y Divulgación de las Ciencias, vol. 19, núm. 1, 2022

Universidad de Cádiz, España

Disponible en: https://www.redalyc.org/articulo.oa?id=92068491009

DOI: https://doi.org/10.25267/Rev_Eureka_ensen_divulg_cienc.2022.v19.i1.1302 
Diseño y evaluación de una intervención educativa sobre la pandemia de la COVID-19 y las medidas de prevención

Design and evaluation of an educational intervention on the COVID-19 pandemic and prevention measures

Ane Portillo-Blanco

Departamento de Física Aplicada, Escuela de Ingeniería de Gipuzkoa, UPV/EHU. Donostia. STEMER-Science, Technology, Engineering and Mathematics Education Research Group, España ane.portillo@ehu.eus

iD https://orcid.org/0000-0002-5439-440X

José Ramón Diez

Departamento de Didáctica de las Matemáticas, Ciencias Experimentales y Sociales, Facultad de Educación de Bilbao, UPV/EHU. Leioa. STEMER-Science, Technology, Engineering and Mathematics Education Research Group, España joseramon.diez@ehu.eus

iD https://orcid.org/0000-0003-3967-0186

Oihana Barrutia

Departamento de Didáctica de las Matemáticas, Ciencias Experimentales y Sociales, Facultad de Educación, Filosofía y Antropología. UPV/EHU. Donostia. STEMER-Science, Technology, Engineering and Mathematics Education

Research Group, España

oihana.barrutia@ehu.eus

iD https://orcid.org/0000-0003-4118-7791

\section{Mikel Garmendia}

Departamento de Expresión Gráfica y Proyectos, Escuela de Ingenieria de Gipuzkoa, UPV/EHU. Donostia STEMERScience, Technology, Engineering and Mathematics

Education Research Group, España

mikel.garmendia@ehu.eus

iD https://orcid.org/0000-0003-1543-5114

Jenaro Guisasola

Departamento de Física Aplicada, Escuela de Ingeniería de Gipuzkoa, UPV/EHU. Donostia. STEMER-Science, Technology, Engineering and Mathematics Education Research Group, España jenaro.guisasola@ehu.eus

(iD https://orcid.org/0000-0005-0817-3905
DOI: https://doi.org/10.25267/

Rev_Eureka_ensen_divulg_cienc.2022.v19.i1.1302 Redalyc: https://www.redalyc.org/articulo.oa? $\mathrm{id}=92068491009$
Recepción: 27 Abril 2021

Revisado: 09 Julio 2021

Aprobación: 13 Octubre 2021 


\title{
RESUMEN:
}

La crisis provocada por la pandemia de la COVID-19 ha llevado a la mayoría de las personas en todo el mundo a realizar un cambio en sus actividades cotidianas y ha resaltado la necesidad de una alfabetización científica. Sin embargo, pocos estudios han presentado intervenciones educativas relacionadas con las medidas preventivas ante la COVID-19. En este estudio, presentamos un taller de divulgación científica sobre las medidas de prevención ante esta pandemia con estudiantes de Secundaria, Bachillerato, Grado en Educación Primaria y Máster en Formación del Profesorado de Secundaria, con el objetivo de investigar sus concepciones y razonamiento sobre las medidas de seguridad frente a la COVID-19. En particular, cómo la información basada en evidencias científicas puede influir en la toma de decisiones en la vida diaria y en la decisión de ponerse o no la vacuna. Mediante un diseño prepost se indaga sobre las ideas y formas de razonar del alumnado en relación con las medidas de prevención para evitar el contagio de la COVID-19. Los resultados muestran que esta intervención educativa mejora el conocimiento de alumnado sobre la base científica e importancia de las medidas de prevención y su actitud hacia la vacunación.

Palabras Clave: problemas socio-científicos, COVID-19, diseño de actividades de enseñanza, intervención educativa, alfabetización científica.

\begin{abstract}
:
The crisis caused by the COVID-19 pandemic has led most people around the world to make a change in their daily activities and has highlighted the need for scientific literacy. However, few studies have presented educational interventions related to preventive measures against COVID-19. In this study, we present a scientific literacy workshop on security measures against COVID-19 with Secondary Education, Upper Secondary Education, BA in Primary Education and Master's Degree in Secondary Teacher Training, with the aim of investigating their conceptions and reasoning about preventive measures against COVID-19. In particular, how information based on scientific evidence can influence decision-making in daily life and the decision to get the vaccine or not. Through a pre-post design, students' ideas and ways of reasoning are investigated in relation to security measures to avoid the spread of COVID-19. The results show that this educational intervention improves students' knowledge about the scientific basis and importance of prevention measures as well as their attitude towards vaccination.
\end{abstract}

KEYWORDS: socio-scientific issues, COVID-19, teaching activity design, instructional intervention, scientific literacy.

\section{INTRODUCCIÓN}

Durante 2020 y 2021 estamos viviendo un período extraordinario, en el que en muchas partes del mundo se han tomado medidas sociales excepcionales para prevenir la enfermedad de la COVID-19 en la población. Asimismo, la pandemia provocada por la expansión del virus SARS-COV-2 ha puesto en evidencia a escala mundial la enorme presencia que tiene la ciencia en la vida cotidiana y, a su vez, la falta de alfabetización científica de la ciudadanía. Esto se ha visto reflejado en la cantidad de bulos que durante todos estos meses se han propagado por las redes sociales y que la gente, sin poner en duda la información, ha difundido rápidamente. Todo ello, ha complicado aún más la tarea de conseguir que se entienda tanto la situación que se está viviendo como las medidas a tomar para prevenirlo (Erduran 2020).

Las dificultades de comunicación entre las organizaciones sanitarias y la ciudadanía pueden ser debidas, en parte, a la escasa alfabetización científica que se adquiere durante la educación obligatoria. El objetivo de la educación científica no es sólo conseguir que el alumnado adquiera unos conocimientos que le permitan avanzar en su recorrido académico, sino también capacitarlo para que participe en discusiones públicas sobre situaciones de interés colectivo y social (Furió et al. 2001). Como explica Reiss (2020) temas importantes relacionados con la historia, la filosofía y la sociología de la ciencia deben formar parte de una educación y alfabetización científica de los estudiantes en la sociedad actual. Asimismo, la educación científica debe desarrollar el pensamiento crítico de cada individuo; es decir, desarrollar una manera de pensar que les permita tomar posiciones argumentadas frente a situaciones sociales relacionadas con la ciencia (Solbes y Torres 2012). Existe un consenso para incluir en la enseñanza de las ciencias temas que permitan entender las implicaciones sociales de la ciencia, incluyendo tanto los aspectos éticos como políticos de esta (Zeidler et al. 2002, Reiss 2020). 
Durante 2020 y 2021 los debates sociales en torno a la pandemia han estado constantemente presentes y, entre otras cuestiones, se ha puesto en duda el origen del virus, las vías de propagación, etc. En este contexto, hemos visto la necesidad y oportunidad de diseñar, implementar y evaluar un taller de divulgación sobre las medidas de higiene y prevención ante la pandemia de la COVID-19 con el objetivo de que los y las estudiantes entiendan los motivos de dichas medidas y el impacto de su cumplimiento en la sociedad. Pensamos que facilitar la comprensión del alumnado sobre las medidas preventivas frente al contagio y los beneficios de la vacuna podría ayudar a la promoción de hábitos saludables.

En consecuencia, decidimos llevar a cabo una intervención en forma de taller de divulgación científica en el contexto académico de niveles educativos de Secundaria, Bachillerato y Universidad, con el objetivo de investigar cuáles eran las ideas de los estudiantes sobre las medidas preventivas frente al contagio de SARS-CoV-2 y cómo la experiencia del taller podía ayudar a comprender la base científica de dichas medidas. El taller integra cuatro problemas fundamentales del desarrollo de la pandemia y su reflejo en las correspondientes medidas de prevención ante el contagio y la fabricación de una vacuna. El análisis de estos problemas motiva y profundiza la comprensión de la pandemia por parte del estudiantado, mientras que los avances tecnológicos de fabricación de mascarillas y vacunas les permiten visualizar las motivaciones de las medidas propuestas por las organizaciones internacionales de salud. Por otra parte, este trabajo pretende contribuir a la necesidad de información sobre el efecto que talleres divulgativos de corta duración en el tiempo tienen sobre el alumnado de diferentes niveles educativos. En el estudio evaluamos si el formato de taller como intervención educativa puntual en el proceso de enseñanza mejora la capacidad del alumnado para comprender la pandemia y las medidas preventivas propuestas.

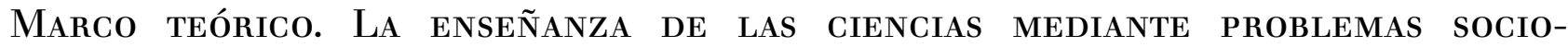 CIENTÍFICOS}

El objetivo principal que guía esta investigación es indagar sobre qué opiniones expresa el alumnado de diferentes etapas educativas y qué decisiones toma frente a la pandemia provocada por la COVID-19 cuando se enfrentan a información basada en evidencias científicas y que puede desafiar sus creencias iniciales. Este objetivo se desarrolló en el marco teórico de enseñanza mediante problemas socio-científicos, el cual parte de la convicción de que involucrar a los y las estudiantes en actividades socio-científicas que requieran un pensamiento crítico puede llevar al alumnado a una mejor comprensión de las prácticas científicas y sus relaciones con problemas sociales (Zeidler et al. 2002).

La enseñanza mediante problemas socio-científicos busca un aprendizaje contextualizado en el que se dé importancia al contexto social y cultural en el que se desarrolla la ciencia y, para conseguirlo, el alumnado debe conocer sus procedimientos, normas y formas de actuar propias (Sadler 2009). Así, como explican Brown et al. (1989), el alumnado debe aprender mediante actividades reales para poder ver cada situación desde el punto de vista científico. Estas propuestas educativas se caracterizan por trabajar problemas sociales actuales, controversiales e interesantes para el alumnado y que además están relacionados con la ciencia y abren la opción a debatir (Hancock et al. 2019). Del mismo modo, tienen varias soluciones y no pueden resolverse utilizando solo contenidos científicos simples y memorizados. En este sentido, diversos estudios han demostrado que la enseñanza basada en problemas socio-científicos tiene un impacto positivo en el aprendizaje del contenido de ciencias (Herman 2015), en la comprensión de la naturaleza de la ciencia (Zeidler et al. 2002) y en la capacidad de argumentación y análisis del alumnado (Zeidler et al. 2013). En resumen, que la enseñanza-aprendizaje basada en problemas socio-científicos ha demostrado ser una manera efectiva de contextualizar el conocimiento científico dentro de un contexto social complejo (Zeidler 2014, Hancock et al. 2019).

Otro elemento teórico importante que guía este trabajo es la asunción de que en la toma de decisiones frente a problemas socio-científicos influye el llamado razonamiento emocional. Este razonamiento 
considera que las personas pueden rechazar incluso la evidencia científica cuando colisiona con sus ideas religiosas o ideológicas. La investigación en psicología cognitiva se refiere al "razonamiento motivado" como aquel que lleva a las personas a rechazar o ignorar la evidencia para proteger sus valores, actitudes y visiones del mundo (Sinatra et al. 2014). McIntyre (2019) defiende que, a la hora de presentar la información y el debate en el aula, se ha de considerar que las personas pueden presentar sesgos cognitivos que les convencen de que sus conclusiones se basan en razonamientos sólidos, mientras que las opiniones de los demás son falaces. Estos sesgos cognitivos relacionados con las emociones y el aprendizaje han sido ampliamente investigados en la enseñanza de las ciencias, revelando su importancia a la hora de aprender y enseñar (Costillo Borrego et al. 2013, Mellado Jiménez et al. 2014).

A la hora de elegir como tema socio-científico las medidas preventivas frente a la pandemia de la COVID-19, hemos tenido en cuenta que uno de los principales requisitos que establece la enseñanza mediante problemas socio-científicos a la hora de elegir un tema es que sea interesante para el alumnado. Según Stolz et al. (2013) un problema será relevante si afecta a su vida actual o futura, por lo que el escenario que nos presenta la pandemia de la COVID-19 encaja a la perfección en esta característica. Además, el conocimiento que tiene la sociedad sobre el tema es escaso y la cantidad de bulos e información manipulada que hay en los medios de comunicación y redes sociales puede tener un impacto negativo en la percepción que tiene la gente sobre la pandemia (van Prooijen 2017, Okunlola 2020).

En este contexto de tratamiento de problemas socio-científicos relacionados con la COVID-19, existen estudios previos que muestran las implicaciones de factores diferentes a los estrictamente científicos. Así, un estudio previo realizado por Roozenbeek et al. (2020) analizó la relación entre la susceptibilidad a la desinformación sobre la COVID-19 y el cumplimiento de las medidas de seguridad y la intención de vacunación. En sus resultados mostraron que el aumento de la susceptibilidad iba unido con una menor intención de vacunación y de cumplimiento de las medidas y que, a su vez, esa susceptibilidad aumentaba a medida que las personas desconfiaban más en la ciencia. Siguiendo la misma tendencia, otro estudio previo relacionó el nivel académico con la creencia en teorías conspiratorias relacionadas con la COVID-19, ya que el hecho de creer en ellas suele ir unido con un rechazo a las medidas de prevención, tratamientos médicos y vacunas (Georgiou, Delfabbro y Balzan 2020). Estos resultados convergen con los de Zeidler et al. (2002) que muestran que cuanto mejor es la comprensión de la naturaleza de la ciencia, mayor es el enfoque científico con el que analizan los aspectos ético-morales del tema. Estos estudios previos que implican razonamiento emocional, formación académica y susceptibilidad se tuvieron en cuenta a la hora de diseñar algunas preguntas del taller, que incidían además de en su conocimiento científico sobre el tema, en el ámbito personal y de creencias de los y las participantes (ver tabla de preguntas en el Anexo). Así mismo, se ha tenido en cuenta que existen estudios previos sobre las dificultades de aprendizaje de algunos de los contenidos científicos tratados en el taller que nos indican que su enseñanza muestra en algunos casos especial resistencia a su aprendizaje a lo largo de las etapas educativas. Por ejemplo, se han analizado las dificultades del alumnado a la hora de entender la diferencia del tamaño del virus y de las partículas (Benarroch 2001, Treagust et al. 2010, AAAS Science Assesment 2021) así como los fenómenos de difusión (Tang et al. 2021, Salter 2021).

De acuerdo con los elementos teóricos expuestos, en el diseño del taller que presentamos se asume que la presentación de problemas socio-científicos relativos a la pandemia de la COVID-19 ayuda a una alfabetización científica. Esto es, el taller pretende generar en los y las estudiantes actitudes positivas hacia las medidas de prevención frente a la pandemia, mediante la comprensión contextualizada de las ideas clave de los aspectos más relevantes de la pandemia y de las medidas de prevención adoptadas por las organizaciones sanitarias. 


\section{Diseño DEL TALLER: UN PROCESO INTERACTIVO DE PRESENTACIÓN DE PROBLEMAS SOCIO- CIENTÍFICOS}

El taller se diseñó a partir de un análisis de la información de las medidas preventivas sobre la pandemia en los medios de comunicación y las organizaciones de promoción de la Salud, así como de los argumentos científicos básicos que permiten entender dichas informaciones. En particular, el taller se secuenció en cuatro grandes problemas socio-científicos: el uso de la mascarilla, la aplicación del distanciamiento social, la conveniencia de la ventilación y la eficacia de la vacuna. Desde el comienzo de la pandemia, las medidas a las que más importancia se les ha dado desde el Ministerio de Salud y los medios de comunicación han sido la mascarilla y la distancia social. Asimismo, el uso de la mascarilla ha generado un debate social acompañado de varios bulos que creemos que era indispensable discutir, por lo que, la primera medida a analizar en el taller fue el uso de la mascarilla. Por otra parte, desde las organizaciones de Salud se insiste en que las mascarillas no evitan el contagio en todos los casos y en que se necesitan más medidas preventivas frente a la propagación del virus, así que la siguiente medida que se trabajó fue la distancia social (Galbadage, Peterson y Gunasekera 2020).

Otra medida de prevención frecuentemente comentada es la ventilación para evitar el riesgo de contagio por aerosoles, que traspasan la mascarilla y recorren distancias largas (Morawska et al. 2020). Además, se realizó especial hincapié en la importancia de combinar las medidas de prevención para conseguir la mayor seguridad posible.

Del mismo modo, no podíamos ignorar el debate social creado en torno a las vacunas desde el comienzo de la pandemia, así como la cantidad de ideas erróneas que se han propagado. Por ello, añadimos este problema socio-científico a los tres anteriores, aunque su tratamiento varió de los talleres realizados en diciembre de 2020 (Secundaria y Bachillerato) a los realizados con posterioridad (Grado y Máster). La razón es que la vacunación en España comenzó a finales de diciembre de 2020 y que los artículos científicos relativos a las vacunas de Pfizer-BioNTech y Moderna se publicaron el 31 de diciembre de 2020 y 4 de enero de 2021 respectivamente (Polack et al. 2020, Baden et al. 2021).

De acuerdo con lo anterior, el taller se construye como un ciclo de resolución de estos cuatro problemas socio-científicos para desarrollar explicaciones conceptuales cada vez más complejas e ideas interconectadas que describen o explican una versión simplificada del fenómeno. A continuación (Figura 1) mostramos la relación entre los cuatro problemas guía, donde el análisis de cada una de las medidas y sus carencias nos lleva a la siguiente, interconectándolas y dejando al descubierto la necesidad de cumplirlas en conjunto. Se trata de ayudar a los y las estudiantes a desarrollar representaciones que describen o explican una versión simplificada del fenómeno que se puede utilizar para hacer predicciones (Garmendia y Guisasola 2015). 


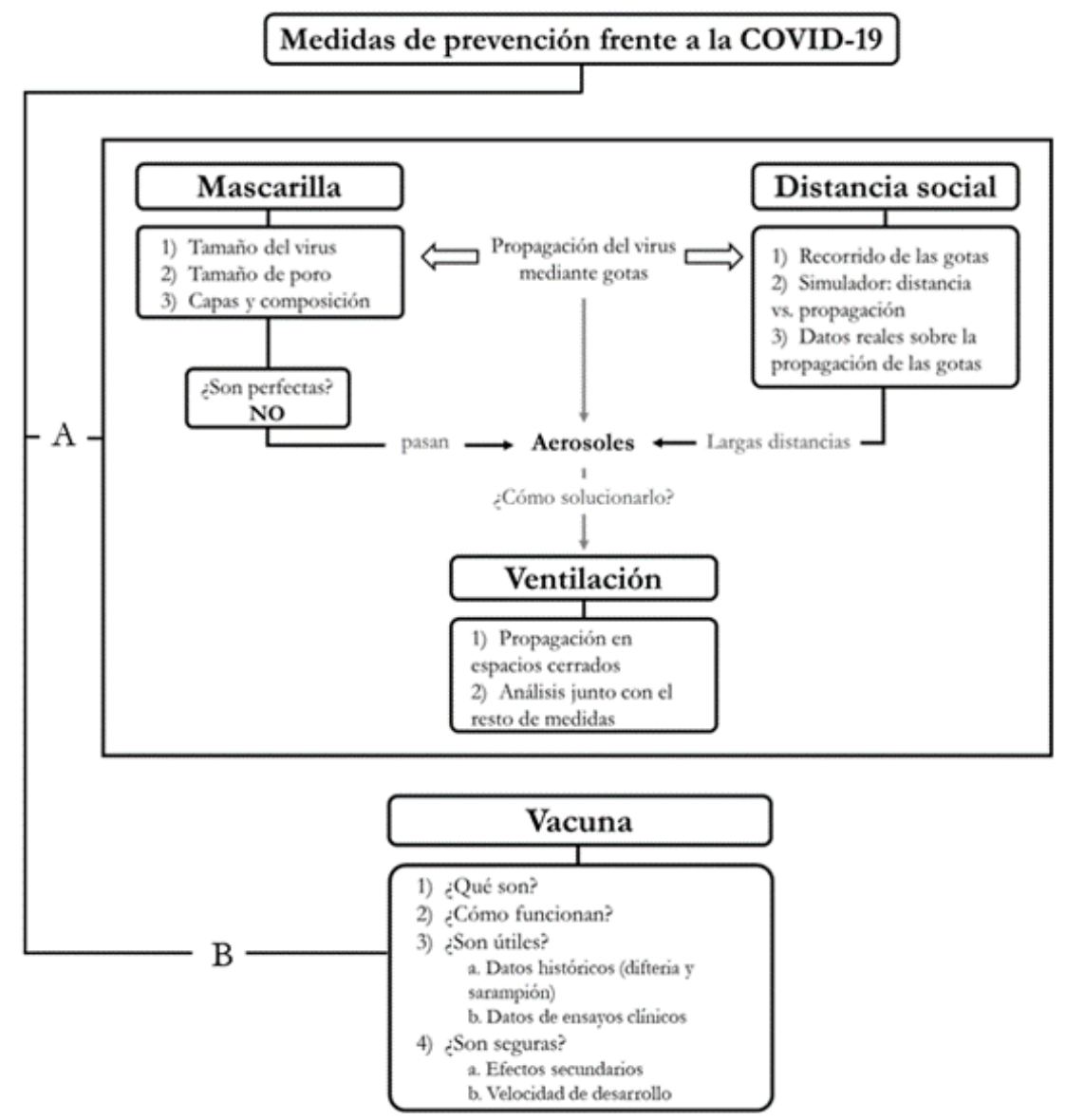

FIGURA 1

Conexión entre los cuatro problemas sociocientíficos sobre prevención frente a la COVID19 trabajados durante el taller A Medidas de prevención físicas B Medidas de prevención inmunológicas

El taller comenzó con una invitación a los y las estudiantes para que escribieran en un minuto todas las medidas de prevención contra la COVID-19 que conocían (cuestión Q1, ver Anexo I con todas las cuestiones del taller) para indagar sobre las medidas más conocidas por el alumnado. A continuación, se abordó la mascarilla como medida de prevención. La pregunta inicial está diseñada para que el alumnado argumente sobre su uso, ventajas y desventajas (cuestión Q2). Si el objetivo de la mascarilla es evitar solo el traspaso del virus, la clave de su funcionamiento debe radicar en la diferencia de tamaño de los gases que inhalamos y exhalamos frente al tamaño de virus y gotículas que los transportan. Para ayudar al alumnado a concretar sus ideas sobre el tamaño de las moléculas de $\mathrm{CO}_{2}$ y $\mathrm{O}_{2}$, de un virus, de una bacteria y de una célula humana, se diseñó la cuestión Q3, que sirve como motivación para comprender que conocer el tamaño de esos elementos es imprescindible para poder definir posteriormente el tamaño de poro de la mascarilla (cuestiones Q4 y Q5). A continuación, se debatió (cuestión Q6) y analizó la diferencia entre una mascarilla de tela y una quirúrgica mediante imágenes de Neupane et al. (2019) y Verma, Dhanak y Frankenfield (2020) y mediante el análisis de un vídeo que comparaba la capacidad de filtración de las gotículas en el momento de hablar, toser y estornudar a través de distintas mascarillas y en ausencia de mascarilla (Bahl et al. 2020). Este último vídeo llevaba al alumnado a concluir que las mascarillas quirúrgicas, a pesar de ser mejores que las de tela, no son perfectas y de este modo, se comenzaba a trabajar la idea de que con respetar una sola medida no es suficiente (Figura 2). 


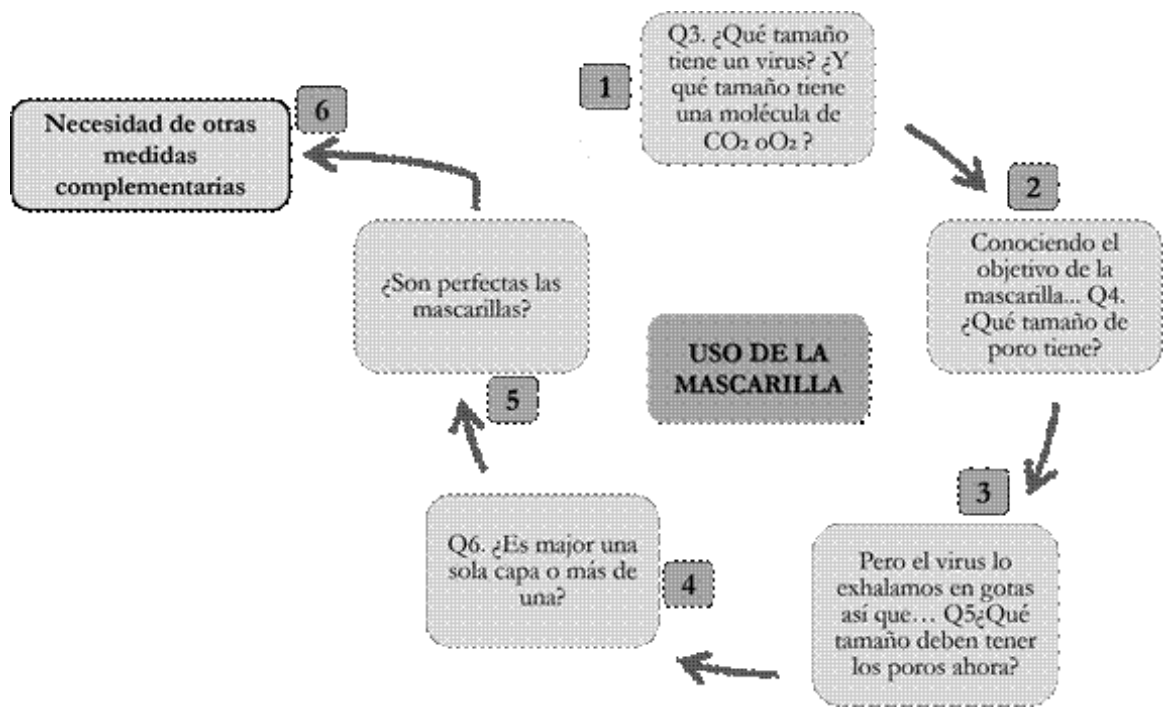

FIGURA 2

Ciclo de actividades sobre el problema "uso de la mascarilla".

La siguiente medida que se trabajó en el taller fue la distancia social (Figura 1) y para ello se comenzó con un desafío (cuestión Q7) para saber a qué distancia creía el alumnado que se evita el riesgo de contagio. Para proporcionar argumentos basados en evidencias científicas se pasó a analizar una simulación y unos datos empíricos obtenidos de las investigaciones sobre el tema (Galbadage, Peterson y Gunasekera 2020, Morawska et al. 2020). Los resultados empíricos ayudan a que los y las estudiantes vean de forma gráfica que cuanta mayor distancia mantenemos menor es la probabilidad de contagio y la velocidad de propagación del virus. Este hecho se aprovechó para concluir que no hay una distancia que sea completamente segura, ya que los aerosoles (las gotas de menor tamaño) recorren largas distancias. Así que necesitamos una medida más y esa es precisamente la ventilación (Figura 1).

Se inició el apartado de la ventilación con una pregunta desafío (cuestión Q8) que indaga sobre qué medida creen que se debería utilizar para minimizar el contagio mediante aerosoles. Para el análisis de esta medida de prevención se utilizaron varios vídeos en los que, por un lado, se veía cómo a medida que pasamos más tiempo en un espacio cerrado aumenta la concentración de aerosoles y por lo tanto el riesgo de contagio. Por otro, se utiliza la iconografía de un aula para ver cómo afecta la ventilación en la propagación entre las personas presentes (El País 2020).

En cuanto al último problema guía, en los talleres realizados en diciembre 2020, se utilizaron datos de vacunas aplicadas durante el siglo XX (la vacuna de la difteria y la triple vírica) para ver la evolución de casos de dichas enfermedades (Isabella 2015) y, en los talleres en enero-febrero 2021 se añadieron los datos publicados de los ensayos clínicos de Pfizer-BioNTech y Moderna (Polack et al. 2020, Baden et al. 2021). La exposición y discusión de los datos permite que el alumnado reflexione sobre el efecto que han tenido y siguen teniendo las vacunas que son administradas, ya que la mayoría del alumnado desconoce muchas de esas enfermedades frente a las que somos inmunizados durante nuestra infancia. Asimismo, los datos aportados y la exposición sobre cómo se obtienen las vacunas contra la COVID-19 permiten que el estudiantado tome conciencia del tamaño de muestra utilizada, de cómo funciona el doble ciego que se utiliza para analizar su efectividad y de la diferencia de casos positivos registrados en cada grupo. Es decir, se reflexiona sobre cómo se utilizan los procedimientos científicos y la cooperación en la interface ciencia-sociedad (Zeidler et al. 2002). 


\section{Metodología}

\section{Participantes}

Este taller se ha llevado a cabo con 828 estudiantes de Enseñanza Secundaria Obligatoria (ESO), Bachillerato, Grado en Educación Primaria y Máster de Formación del Profesorado de Secundaria. La selección de la muestra se hizo de acuerdo con el objetivo del estudio de realizar una intervención de alfabetización científica en niveles educativos desde Secundaria Obligatoria hasta Universidad y posgrado. Participaron en el taller 485 personas de ESO y 112 de Bachillerato, todas pertenecientes a tres centros concertados de secundaria del País Vasco. En el caso del alumnado universitario, todos cursaban estudios en la Universidad del País Vasco/Euskal Herriko Unibertsitatea (UPV/EHU), y participaron 176 estudiantes de primer curso del Grado de Educación Primaria y 55 estudiantes del Máster de Formación de Profesorado de Secundaria en las especialidades de Tecnología y de Ciencias Naturales y Matemáticas. Debido a las condiciones de docencia en la Comunidad Autónoma Vasca, el taller se realizó con todos los y las estudiantes de forma presencial.

En el currículo educativo de la Comunidad Autónoma Vasca (Heziberri 2020) los contenidos que más pueden relacionarse con la pandemia de la COVID-19 se dan en $3^{\circ}$ de ESO (14-15 años) en las asignaturas de Biología y Geología y en Física y Química, donde se trabaja la salud, el sistema inmunitario y la diferencia de tamaño entre una molécula, un virus, una bacteria y una célula del cuerpo humano (célula eucariota). Así pues, el alumnado a partir de $3^{\circ}$ de ESO debería conocer los contenidos conceptuales básicos que se tratan en el taller. Aun así, hay que tener en cuenta que este curso también es el último en el que las asignaturas de ciencias son obligatorias, por lo que en el caso de las personas que decidan alejarse de las ciencias, esa habrá sido la única vez que hayan tratado con dichos contenidos en su educación. También hay que tener en cuenta que el alumnado de Máster que ha participado en el taller ha estudiado Grados científico-tecnológicos, así que su conocimiento sobre algunos de los contenidos del taller es mucho mayor que en el resto de los y las estudiantes.

\section{El estudio}

El taller se aplicó en cada centro educativo de acuerdo con el profesorado, en una sesión de hora y media para todos los niveles. A la hora de realizar esta intervención, se consideró necesario poder recibir el feedback continuo de todo el alumnado y obtener un registro detallado de sus respuestas, y para ello se utilizó la aplicación web Wooclap. Así, todo el estudiantado utilizó ordenadores portátiles o sus teléfonos móviles. Además, Wooclap permite insertar preguntas de diferentes tipos durante la presentación para que los y las estudiantes respondan al momento (Anexo I). A medida que responden, los resultados aparecen de forma anónima en la pantalla principal, lo que facilita dirigir un debate general en torno a sus respuestas y poder insertar las preguntas a medida que avanza el taller ayuda también a mantener la atención del alumnado.

\section{Evaluación}

Una vez elaborado el cuestionario del Anexo I, realizamos un borrador de prueba con estudiantes de lero y $4^{\circ}$ de ESO, que confirmó que el alumnado no tenía ningún problema en entender cómo se formulaban las preguntas. Indujimos que no habría problemas en estudiantes de cursos superiores. Además, los objetivos de cada pregunta fueron validados por seis profesores (tres de Didáctica de las Ciencias y tres de Biología) que coincidieron con los contenidos y objetivos de las preguntas. Se sugirieron algunos cambios de redacción que fueron realizados. Las preguntas del Anexo I son las definitivas que se pasaron en los talleres. 
Las respuestas de los y las estudiantes a las preguntas de explicación de las respuestas cerradas (Q10, Q12, Q14 y Q16) fueron sometidas a análisis fenomenográfico (Marton 1981). Esto supuso un proceso de categorización de las respuestas del alumnado y una posterior clasificación de las mismas. En este proceso participaron 3 personas del equipo para llegar a un nivel de confianza de la clasificación adecuado para el estudio. La atención se centró en la comprensión del alumnado, tomando las respuestas de los y las estudiantes como un todo, más que en la ocurrencia de declaraciones particulares correspondientes a una categoría específica de descripción. Se utilizó un proceso iterativo para producir descripciones de categorías finales que reflejaran una comprensión similar entre las respuestas asignadas a cada categoría y las diferencias entre las categorías.

Este estudio comprendió un diseño pre-post (Romine et al. 2017), donde se identifican las opiniones sobre medidas preventivas frente a la COVID-19 en relación a los cuatro temas tratados, antes (cuestiones Q1, Q2, Q3, Q4, Q6, Q7, Q8 del anexo I) y después (cuestiones Q5, Q9, Q10, Q11, Q12, Q14 del anexo I) de realizar las tareas. Para la comparación entre resultados pre-test y post-test se ha utilizado el test estadístico de McNemar, que permite el análisis de pruebas no paramétricas dicotómicas relacionadas (Lachenbruch 2014).

El diseño no contempla grupo de control, pero en relación con algunos de los problemas planteados (p. ej. la actitud hacia la vacunación) hemos podido comparar nuestros resultados con los obtenidos en encuestas realizadas a la población española.

\section{Resultados}

\section{¿Qué medidas de seguridad conocen?}

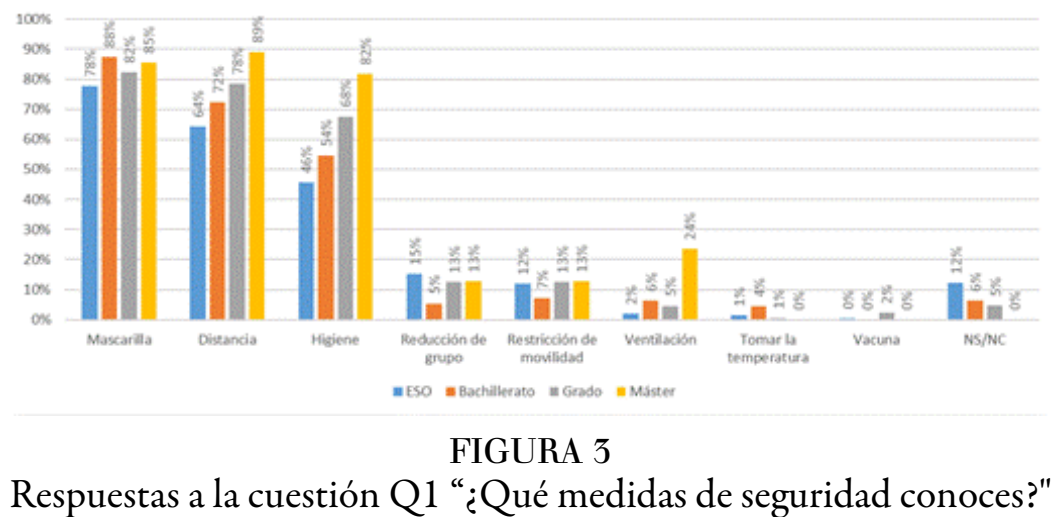

En los cuatro grupos encuestados las tres medidas más mencionadas en la cuestión Q1 fueron el uso de la mascarilla, el distanciamiento social y la higiene (Figura 3). El porcentaje de las tres medidas aumentó con el nivel educativo. Sin embargo, el porcentaje disminuyó al hablar de la restricción de aforo y movilidad, y fue aún menor en el caso de la ventilación excepto en el nivel educativo del Máster. En este caso fue mencionado por la cuarta parte del alumnado.

\section{¿Por qué debemos usar la mascarilla? ¿Qué características tiene?}

Centrándonos en argumentos científicos empleados por el alumnado sobre la efectividad de las mascarillas en relación al tamaño del poro (cuestiones Q4 y Q5), se observa que el porcentaje de respuestas correctas a las cuestiones Q4 (pre-test) y Q5 (post-test) mejora significativamente tras la discusión en el taller (utilizando el test de McNemar $\mathrm{p}<0.001)$. Es decir, después de la discusión sobre el tamaño del poro en la mascarilla, aumenta significativamente el porcentaje de respuestas que explican que las moléculas de $\mathrm{CO}_{2}$ y $\mathrm{O}_{2}$ son de 
menor tamaño que el virus y que los poros son lo suficientemente grandes para dejar pasar a esas moléculas, pero menores que las gotas que transportan el virus, evitando así el contagio.

La efectividad del tamaño del poro de la mascarilla en relación al tamaño del virus fue evaluada en la cuestión Q3 (pre-test) y en las cuestiones Q9 y Q10 (post-test). Al comenzar el taller (cuestión Q3) el porcentaje de estudiantes que tenía conocimiento adecuado del tamaño del virus respecto a otras partículas implicadas en el proceso es muy variable (primera columna en la Figura 4). Después de tratar el tema en el taller, en todos los grupos encuestados, por lo menos el $80 \%$ del alumnado respondió en la cuestión Q9 que el tamaño de poro de la mascarilla influye en la protección que ofrecen. Además, más de la mitad del alumnado argumenta correctamente en la cuestión Q10 diciendo que dependiendo del tamaño del poro el virus o las gotas que lo transportan podrán traspasar la mascarilla o no (segunda columna en la Figura 4).

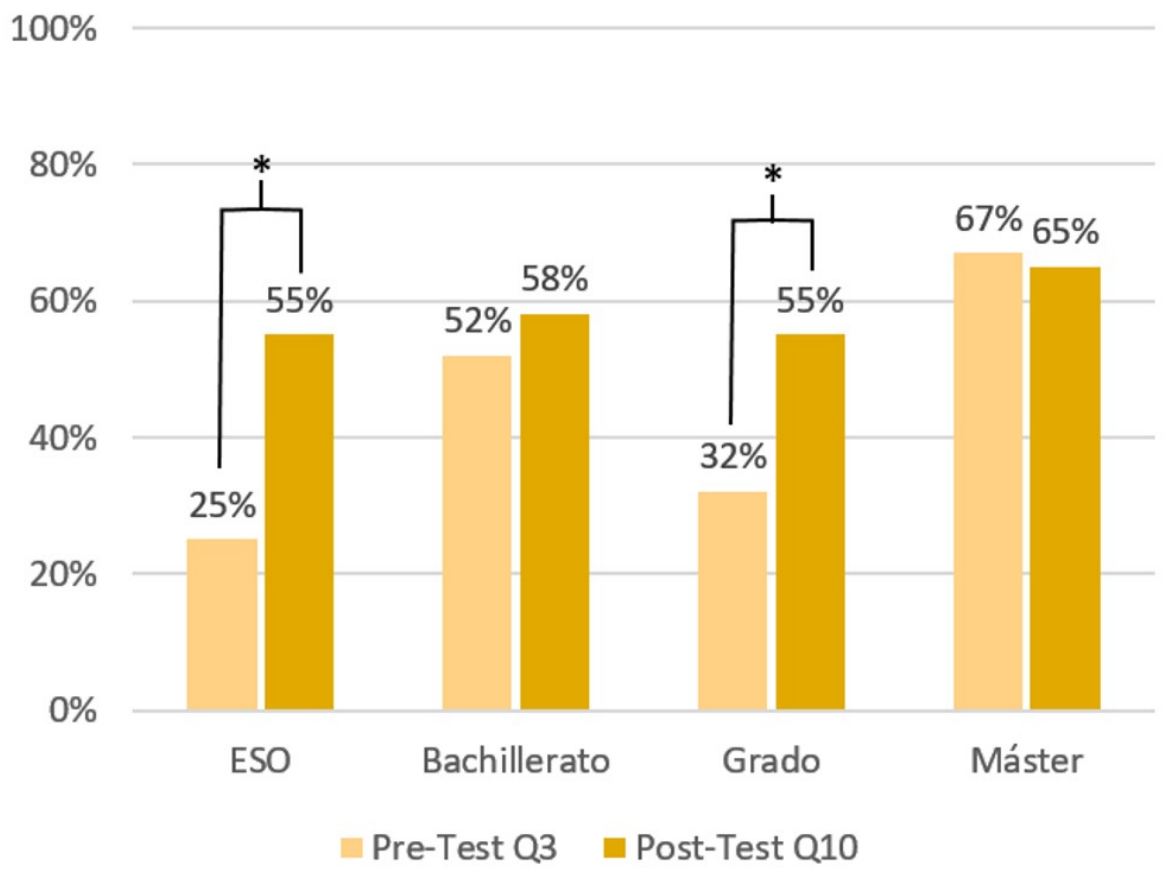

FIGURA 4

Comparación pre-post test de las cuestiones Q3 y Q10. Q3 hace referencia al alumnado que responde que $\mathrm{CO}_{2}$ y $\mathrm{O}_{2}$ son de menor tamaño y $\mathrm{Q} 10$ hace referencia al porcentaje de estudiantes que argumenta que dependiendo del tamaño de poro el virus puede pasar o no. Estadística: Test de McNemar, p < 0,001 (*)

$\mathrm{Al}$ realizar la comparación pre-test post-test entre las cuestiones Q3 y Q10 (Figura 4), encontramos que estas diferencias son estadísticamente significativas en el grupo de ESO y de Grado. En estos grupos también se detecta un mayor desconocimiento previo sobre la diferencia de tamaño de moléculas respecto al virus.

\section{¿Por qué debemos mantener la distancia social y la ventilación?}

Al comienzo del taller alrededor del 70 \% del estudiantado de ESO, Bachillerato y Grado (cuestión Q7) opinó que una distancia completamente segura se encuentra entre 1,5 y $2 \mathrm{~m}$ y no tuvo en cuenta la ventilación (cuestión Q8). Lo que puede producir una sensación de falsa seguridad cuando las personas se encuentran en espacios cerrados durante periodos de tiempo prolongados, creyendo que manteniendo dicha distancia y con la mascarilla puesta el riesgo desaparece. 


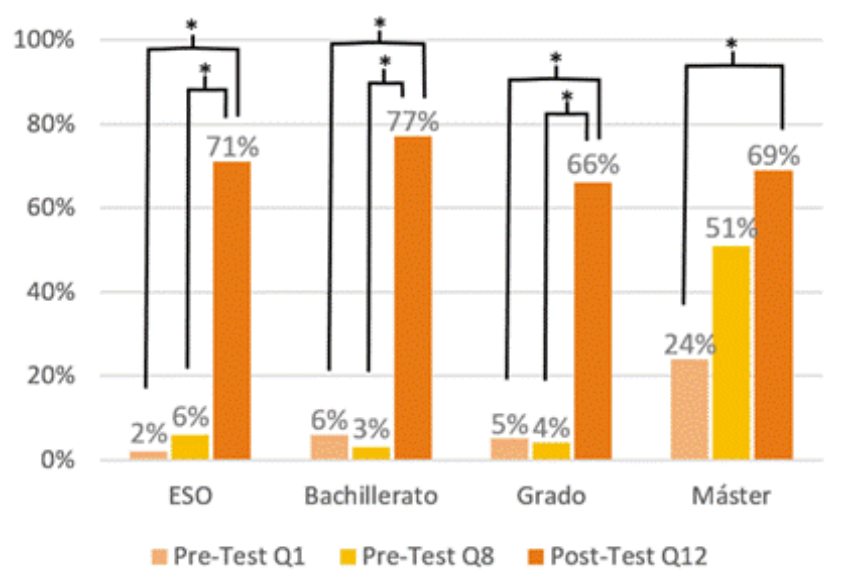

FIGURA 5

Comparación pre-post test sobre la ventilación entre Q1 y Q12 y Q8 y Q12. Estadística: Test de McNemar, $\mathrm{p}<0,001\left(^{*}\right)$

Al final del taller se preguntó por las medidas de seguridad y la necesidad de la ventilación mediante dos cuestiones post-test (cuestiones Q11 y Q12). Las respuestas a la cuestión Q11 mostraron que el 87 \% del alumnado de ESO, el 93 \% de Bachillerato, el $79 \%$ de Grado y el 89 \% de Máster identifican que, en los espacios cerrados además del resto de medidas, es necesario tener la habitación ventilada para evitar el contagio. Del mismo modo, para analizar si el taller ha ayudado a mejorar los conocimientos y la argumentación del estudiantado, se han comparado la cuestión Q1 y Q8 con la argumentación realizada por los estudiantes (cuestión Q12) para saber si hablaban del efecto de la ventilación (Figura 5).

Todas las mejoras son estadísticamente significativas excepto la comparación Q8-Q12 en el grupo del máster, así que podemos afirmar que el taller ha servido para concienciar al alumnado sobre los límites de las medidas del uso de la mascarilla, de la distancia y sobre el impacto de la ventilación en el riesgo de contagio. Asimismo, la mejora en el grupo del máster consideramos que ha sido menor por su formación científica anterior.

\section{¿Por qué debemos ponernos la vacuna?}

En cuanto a la opinión sobre la vacunación, en la cuestión Q13 se aprecia un incremento del porcentaje del alumnado que se vacunaría sin dudarlo en los talleres de enero-febrero respecto a diciembre (Figura 6). Del mismo modo, disminuyen los participantes que prefieren esperar. Por otro lado, si analizamos en conjunto las respuestas de la pregunta de test y los argumentos dados (cuestiones Q13 y Q14), vemos que la principal razón para vacunarse se basa en la confianza en el proceso científico, en los experimentos realizados y en los resultados obtenidos. Así encontramos argumentos como "Yo me pondría la vacuna porque han investigado mucho al respecto y así podré protegerme a mí mismo y a los que me rodean. Además, si nos vacunamos el brote irá disminuyendo y habrá más tiempo para investigar y buscar más soluciones" o "Vacunarme hará que esté protegido de enfermar y no pondré en riesgo a las personas de mi entorno. Además, confío en el trabajo del desarrollo de vacunas y no tengo ningún problema con la vacunación”. En cambio, las personas que dicen que prefieren esperar o directamente no vacunarse, basan sus argumentos en un principio de precaución fundamentado en creencias personales, pero en ningún caso se han encontrado respuestas con una base científica en contra de la vacuna. Así, dentro de ese último grupo encontramos frases como "como es algo nuevo, me da miedo", "las vacunas no sirven y pueden ser perjudiciales para la salud" y "todavía no he visto en la tele o en las revistas que la vacuna sea $100 \%$ segura”. 


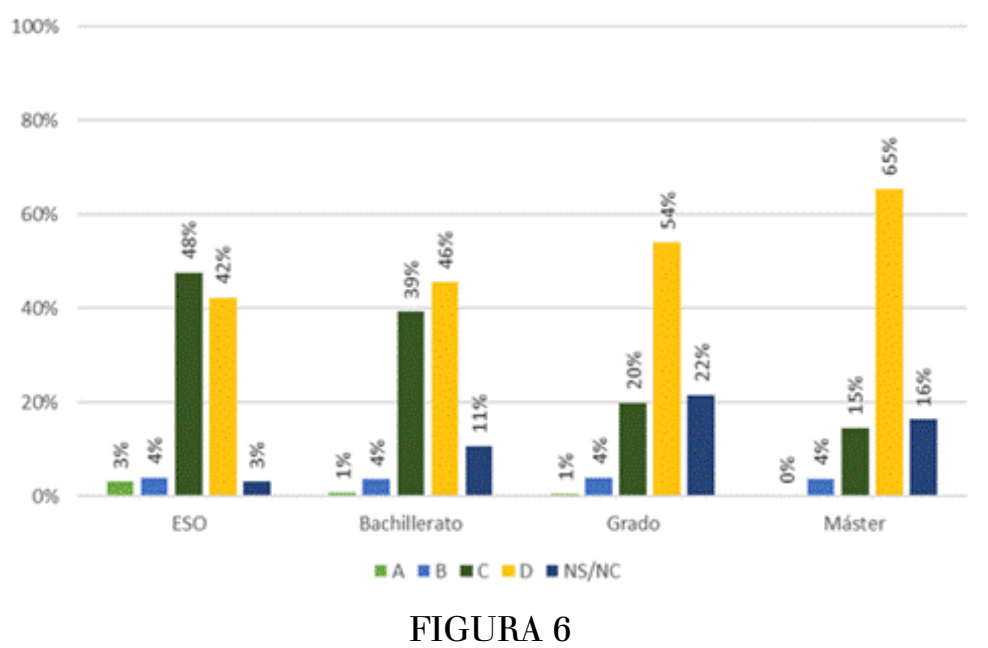

Respuestas del alumnado a la cuestión Q13. A) No, que yo no me la ponga no tiene ningún efecto en la sociedad y no es una enfermedad peligrosa para mi. B) No, prefiero desarrollar inmunidad padeciendo la enfermedad. C) Si, pero no al principio, cuando esté seguro de que funciona. D) Si, porque la vacuna ha seguido un largo proceso de investigación para probar que es efectiva y segura.

Si tenemos en cuenta la encuesta del Centro de Investigaciones Sociológicas (CIS) publicada en diciembre (CIS 2020), sólo el $21 \%$ de las personas se vacunarían sin dudarlo, el $72 \%$ preferiría esperar y el $3 \%$ no se vacunaría. Si tomamos estos porcentajes como un grupo que no ha recibido la información complementaria que se proporciona en el taller y hacemos una comparación con los talleres realizados en diciembre (ESO y Bachillerato), las respuestas son más favorables hacia la vacunación que las que obtiene la encuesta del CIS, por lo que podríamos decir que la información dada ha ayudado a que aumente la confianza hacia las vacunas en el alumnado. Además, si ese aumento va acompañado, como es en nuestro caso, de argumentos basados en el proceso científico de desarrollo de las vacunas y en los datos empíricos ofrecidos en el taller, parece que será más difícil que los bulos sobre vacunas afecten en sus decisiones.

En cambio, en el caso de los talleres de enero-febrero (Grado y Máster), la encuesta de FECYT (Lobera y Cabrera 2021) publicada en febrero reveló que el 58 \% de los encuestados se vacunaría sin dudarlo, el $32 \%$ preferiría esperar y el $9 \%$ no se vacunaría. Estos resultados son similares a los obtenidos en el taller, aunque se aprecia una leve mejora al disminuir el porcentaje de alumnado que preferiría esperar. En el caso del Máster, aumenta el de las personas que se vacunarían ya mismo.

\section{¿Qué medidas de seguridad respeta el alumnado en su día a día?}

La última pregunta del taller tenía como objetivo conocer si el alumnado cumple o no las medidas y por qué actúan de esa manera. Al analizar las respuestas de test (cuestión Q15) como la argumentación (cuestión Q16) se ha repetido el mismo patrón de respuesta en el alumnado de diferentes edades (Figura 7). No obstante, para poder observar el efecto del factor edad en las actitudes hacia las medidas de seguridad, se han analizado las respuestas del alumnado de ESO por cursos en estas dos cuestiones. 


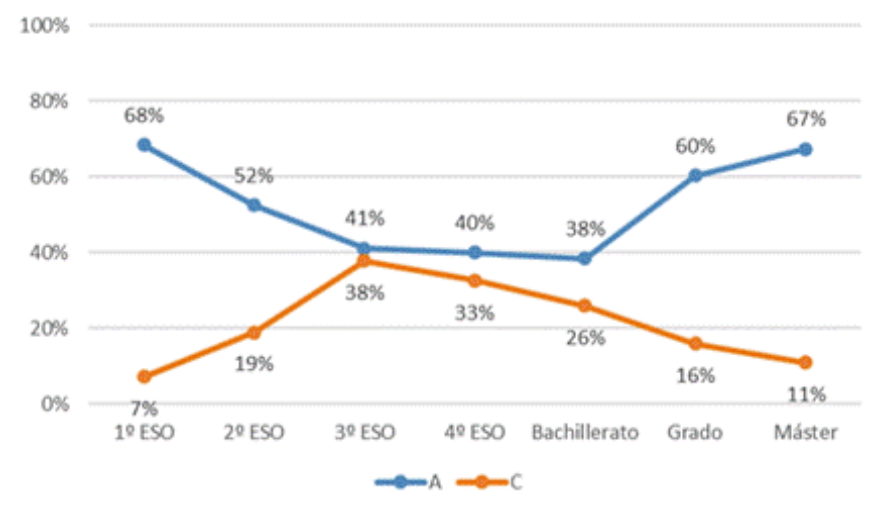

FIGURA 7

Respuestas del alumnado a la cuestión Q15. A) Cumplo las medidas en todas las situaciones posibles. C) Respeto las medidas con las personas mayores, pero cuando estoy con mis amigos y amigas no les damos tanta importancia.

Así, a medida que disminuye el porcentaje del alumnado que dice respetar siempre las medidas, aumenta el de los y las estudiantes que indican no cumplirlas con su grupo de amigos y amigas. El punto de inflexión para ambas cuestiones se encuentra en $3^{\circ}$ de ESO y se mantiene hasta Bachillerato, donde nuevamente se incrementa el porcentaje del alumnado que dice respetar siempre las medidas y baja el de las que no lo hacen con sus amigos y amigas.

Además, cabe destacar la sensación de seguridad que se genera en un ambiente conocido, ya que hemos obtenido respuestas como "cuando estoy con mis amigos y amigas no las respeto, pero siempre que hay gente desconocida lo hago" y el papel que juegan las experiencias previas al respecto, obteniendo respuestas como "entre los amigos y amigas no le damos demasiada importancia a las medidas porque, como hemos visto, el virus no tiene consecuencias en nosotros. Aun así, cuando estoy con gente mayor intento respetar las medidas" o "yo he vivido el virus de cerca y he visto lo malo que puede llegar a ser, así que respeto las medidas para evitar posibles muertes".

\section{DisCUSIÓN Y PERSPECTIVAS PARA LA ENSEÑANZA DE LAS CIENCIAS}

Debido a la pandemia de la COVID-19 desde las autoridades sanitarias se implantaron medidas de prevención para evitar el contagio de este virus que se transmite mediante las gotas que exhalamos. El problema es que el cumplimiento de estas medidas choca con nuestra forma de vida habitual. Todo ello, lleva a definir la pandemia de la COVID-19 como un problema socio-científico de gran importancia que requiere una adecuada alfabetización científica de la sociedad con la finalidad de ayudar a que tomen decisiones utilizando el conocimiento científico. Para facilitar el acceso a dicho conocimiento, es indispensable hacer llegar a la ciudadanía no solo la idea simple de que cumplir las medidas minimiza el contagio, sino el porqué, puesto que cuantos más argumentos científicos se utilizan sobre un tema, mayor es también la tendencia a tener actitudes más favorables hacia ellas y viceversa (Sinatra 2014).

En la implementación del taller hemos comprobado, mediante las preguntas pre-test, que el conocimiento de la mayoría del alumnado sobre los contenidos básicos para entender el funcionamiento y la importancia de las medidas de seguridad era escaso. Esto nos lleva a pensar que el cumplimiento de las medidas es consecuencia de una imposición sin comprensión del porqué y que, además, la información que se ha podido dar en los medios de comunicación no ha llegado de manera efectiva a las personas participantes en el taller. Esto podemos verlo reflejado en la cantidad de bulos que aparecieron en la cuestión Q2 sobre el uso de la mascarilla y los bajos porcentajes de acierto en la cuestión Q3 sobre la diferencia de tamaños entre las 
moléculas y el virus, puesto que conocer esa diferencia es indispensable para comprender el funcionamiento e impacto de la mascarilla y, asimismo, desmentir los bulos sobre los riesgos de utilizarla.

Los resultados obtenidos en la primera pregunta del taller (cuestión Q1, Figura 3), muestran la baja frecuencia con que se mencionan las medidas relacionadas con la ventilación. Esto puede ser debido, entre otros factores, a las diferencias en el tiempo de anuncio y aplicación de cada medida, ya que, la necesidad de mantener los espacios cerrados bien ventilados ha sido la última en incorporarse (septiembre-octubre de 2020) y teniendo en cuenta los resultados obtenidos en esta pregunta, parece que la mayoría del alumnado no es consciente de esta medida.

En relación al conocimiento previo de la influencia del tamaño del poro en la mascarilla (cuestión Q3, Figura 4), los resultados parecen estar de acuerdo con el aprendizaje logrado en el currículo escolar. Es posible que en el Grado en Educación Primaria el bajo porcentaje de respuestas correctas se deba a que gran parte del alumnado haya olvidado los conceptos trabajados en Secundaria y no hayan tenido ocasión de repasarlo en el primer curso del Grado. Después de discutir el tema en el taller, los resultados indican un aumento significativo del conocimiento sobre el tema en aquellos grupos con bajo conocimiento previo (Figura 4). En los dos grupos de Bachillerato y de Máster, en cambio, no se ha visto una evolución significativa en la argumentación y esto probablemente se deba al periodo de instrucción en ciencias recibido. Además, cabe destacar que tener clara la idea de la relación entre el tamaño de poro y las partículas que pasan o no también es clave para desmentir los bulos e ideas erróneas que recogíamos en la cuestión Q2, así que creemos que el hecho de que más de la mitad del alumnado interiorice esa idea ayudará a la toma de decisiones basadas en evidencias científicas.

Si comparamos las respuestas sobre qué medidas de seguridad conoce el alumnado al comienzo del taller con las respuestas a la encuesta del CIS publicada en diciembre de 2020 para la franja de edad de entre 18 y 24 años, vemos que hay coincidencia. Esto nos permite suponer que nuestra muestra inicial puede ser representativa de la población joven debido a la coincidencia de tendencia de los resultados en esta pregunta. Asimismo, vemos que en la población general se mantiene el mismo problema que el detectado en el taller y es que la ventilación ha pasado desapercibida como medida de prevención contra la COVID-19, quizás debido a su tardía incorporación y al escaso hincapié realizado desde las instituciones.

La comparación pre-test y post-test entre las cuestiones Q1, Q8, Q12 (Figura 5) muestra que el desarrollo del taller proporcionó una mejora significativa en la comprensión de que el uso de la mascarilla y de la distancia social no son suficientes en ambientes cerrados y de que la ventilación es importante. Esto significa que un alto porcentaje del alumnado comprendió que, por un lado, la mascarilla y la distancia no son medidas de seguridad perfectas y, por otro lado, que el virus se propaga en gotas de diferentes tamaños y que los aerosoles (las gotas de menor tamaño) suponen un riesgo de contagio cuando su concentración aumenta en espacios cerrados.

Por otro lado, cabe destacar que el porcentaje de respuestas adecuadas del alumnado del Máster de Formación de Profesorado de Secundaria es en todos los casos mayor que en el resto de los grupos. Esto podría ser consecuencia de la edad, pero, teniendo en cuenta la formación científico-técnica de los estudiantes, el resultado coincide con otros estudios en que una formación científica más amplia repercute de forma directa en el conocimiento y comprensión sobre las medidas de prevención (Gallè et al. 2020, Puspitasari et al. 2020).

En cuanto a la opinión sobre la vacunación, vemos una clara diferencia entre el porcentaje del alumnado que se vacunaría sin dudarlo en los talleres realizados en diciembre (ESO y Bachillerato) y en los de enerofebrero (Grado y Máster) (cuestión Q13, Figura 6). Vemos que hay un aumento de entre el 10 y el 20 $\%$ comparando esos dos grandes grupos, lo cual puede ser debido a varias razones: (i) por un lado, se ha visto que el comienzo de la vacunación ha causado un incremento de la confianza hacia la vacuna en la población general (Page 2021) y (ii) por otro, en las encuestas realizadas en diferentes estudios, el porcentaje de aceptación de la vacuna aumenta cuanto más edad tienen las personas encuestadas (CIS 2020, Lobera y Cabrera 2021, Soares et al. 2021), hecho que también sucede en el taller. Además, no debemos pasar por alto 
que la actitud más positiva se ha registrado en el grupo de estudiantes del Máster y esto podría ser debido a su formación científica, como se ha explicado anteriormente. Esto coincide con diferentes estudios que han relacionado el rechazo hacia la vacuna con un nivel educativo más bajo (Loomba et al. 2020, Soares et al. 2021). Los resultados del taller han mostrado unos porcentajes de confianza más positivos que los registrados por las encuestas del CIS y de FECYT. Esto nos lleva a concluir que una correcta formación sobre la vacunación fomenta una actitud más positiva hacia ella.

En cuanto al cumplimiento de las medidas de seguridad, cada persona toma la decisión de cómo quiere cumplir dichas medidas en base a sus intereses y preocupaciones, y esa decisión depende de muchos factores (Sinatra et al. 2014). Las últimas preguntas del taller (Q16 y Q17) muestran que en plena adolescencia aumenta el porcentaje del alumnado que dice que no cumple las medidas cuando está con sus amistades, y lo argumentan diciendo que prefieren seguir como si no pasase nada con ellos o que les resulta incómodo tener que cumplirlas en esas situaciones. Del mismo modo, como explican Sinatra (2014) y Dryhurst et al. (2020), las experiencias personales y cercanas afectan de forma directa a dicho razonamiento motivado, por lo que será más probable que el alumnado que haya vivido casos graves de la COVID-19 en su entorno más cercano cumpla siempre las medidas de seguridad y que suceda lo contrario con las personas que han pasado la enfermedad de forma asintomática y sin consecuencia alguna.

Respecto a las limitaciones de este estudio, los resultados obtenidos se ciñen a un ámbito concreto (la Comunidad Autónoma Vasca) y en este sentido, los resultados del taller se deben entender con las limitaciones de la muestra. Sin embargo, las tendencias que muestran los resultados son coincidentes con otros estudios sociológicos a nivel español y con los resultados de otros estudios internacionales sobre la necesidad de alfabetización científica de la ciudadanía.

El taller se diseñó como un entorno de aprendizaje para resaltar el hecho de que las medidas preventivas contra la COVID-19 y las vacunas son fruto del razonamiento científico y el consenso crítico entre la comunidad científica. De acuerdo con los resultados, la adquisición de un mayor conocimiento sobre un problema socio-científico fomenta en las personas una actitud más acorde a lo consensuado por la comunidad científica hacia dicho problema. Por lo que, en el contexto de la pandemia de la COVID-19, es indispensable no solo el llamamiento a la ciudadanía a cumplir ciertas medidas de prevención para prevenir los contagios, sino que debemos asegurar una adecuada alfabetización científica. Así, este taller sobre cuestiones socio-científicas relacionadas con la pandemia de la COVID-19 contribuye a que el alumnado desarrolle representaciones científicas más elaboradas ante las medidas preventivas propuestas. En el taller se han discutido los argumentos de los consensos y evitado la impresión de que los acuerdos puedan estar sesgados por una "élite científica" o porque lo dice el profesor/a. Explicar los límites de los modelos científicos y las incertidumbres de la ciencia, ayudan a no considerar las explicaciones científicas como "la verdad" sino como intentos honestos de explicar el mundo natural y mejorar la vida de la ciudadanía. Como está mostrando la pandemia de la COVID-19, el conocimiento científico es imprescindible para poder afrontar con las mejores garantías los problemas socio-científicos y los resultados del taller muestran que el diseño de este tipo de entornos de aprendizaje es efectivo para mejorar la comprensión y el compromiso de los estudiantes con la ciencia. Teniendo esto en cuenta y coincidiendo con las conclusiones de otras investigaciones como Lopes y McKay (2020) o Gray et al. (2020), subrayamos la idea de la importancia de la alfabetización científica como vía para formar a las personas en torno al porqué de las medidas de prevención y de la vacunación y así, fomentar una actitud más positiva hacia ellas.

\section{Materiales SUPlementarios}

Anexo I (pdf) 


\section{Agradecimientos}

Agradecemos el apoyo financiero parcial del proyecto del Gobierno Vasco PIBA-IT1349-19 y del Gobierno Español MINECO PID2019-105172-RB-100. Agradecemos también a las escuelas y profesorado de Betiko Ikastola, Ander Deuna Ikastola y San Nikolas Ikastola por su participación en la implementación de este proyecto.

\section{REFERENCIAS}

AAAS Science Assessment Atoms, Molecules, and States of Matter (s. f.) AAAS Science Assesment. Recuperado 15 de julio de 2021, de http://assessment.aaas.org/topics/1/AM/31\#/2

Baden L. R., El Sahly H. M., Essink B., Kotloff K., Frey S., Novak R., ... Zaks T. (2021) Efficacy and safety of the mRNA-1273 SARS-CoV-2 vaccine. New England Journal of Medicine 384(5), 403-416.

Bahl P., Bhattacharjee S., de Silva C., Chughtai A. A., Doolan C., MacIntyre C. R. (2020) Face coverings and mask to minimise droplet dispersion and aerosolisation: a video case study. Thorax 75(11), 1024-1025.

Benarroch A. B. (2001) Una interpretación del desarrollo cognoscitivo de los alumnos en el área de la naturaleza corpuscular de la materia. Enseñanza de las ciencias: revista de investigación y experiencias didácticas 123-134.

Brown J. S., Collins A., Duguid P. (1989) Situated cognition and the culture of learning. Educational researcher 18(1), $32-42$.

CIS (2020) Efectos y consecuencias del Coronavirus (III). Cruzadas por variables sociodemográficas. Diciembre 2020. https://cutt.ly/tv4sYQQ

Costillo Borrego E., Borrachero Cortés A. B., Brígido Mero M., Mellado Jiménez V. (2013) Las emociones sobre la enseñanza-aprendizaje de las ciencias y las matemáticas de futuros profesores de Secundaria. Revista Eureka sobre enseñanza y divulgación de las ciencias 10 (extra), 514-532.

Dryhurst S., Schneider C. R., Kerr J., Freeman A. L., Recchia G., Van Der Bles A. M., ... van der Linden S. (2020) Risk perceptions of COVID-19 around the world. Journal of Risk Research 23(7-8), 994-1006.

Eastwood J. L., Sadler T. D., Zeidler D. L., Lewis A., Amiri L., Applebaum S. (2012) Contextualizing nature of science instruction in socioscientific issues. International Journal of Science Education 34(15), 2289-2315.

El País (2020) Un salón, un bar y una clase: así contagia el coronavirus en el aire. La crisis del Coronavirus. https:// cutt.ly/Wv4s79T

Erduran S. (2020) Science Education in the Era of a Pandemic. Science \& Education 29(2), 233-235.

Furió C., Guisasola J., Vilches A., Romo V. (2001) Finalidades de la enseñanza de las ciencias en la secundaria obligatoria ¿alfabetización científica o preparación propedéutica? Enseñanza de las ciencias 19(3), 365-376

Galbadage T., Peterson B. M., Gunasekera R. S. (2020). Does COVID-19 Spread Through Droplets Alone? Frontiers in Public Health 8, 163.

Gallè F., Sabella E. A., Da Molin G., De Giglio O., Caggiano G., Di Onofrio V., ... Napoli C. (2020) Understanding knowledge and behaviors related to COVID-19 epidemic in Italian undergraduate students: the EPICO study. International journal of environmental research and public health 17(10), 3481.

Garmendia M., Guisasola J. (2015) Alfabetización científica en contextos escolares: El Proyecto Zientzia Live! Revista Eureka sobre Enseñanza y Divulgación de las Ciencias 12(2), pp-294.

Georgiou N., Delfabbro P., Balzan R. (2020) COVID-19-related conspiracy beliefs and their relationship with perceived stress and pre-existing conspiracy beliefs. Personality and individual differences, 166, 110201.

Gray D. J., Kurscheid J., Mationg M. L., Williams G. M., Gordon C., Kelly M., ... McManus D. P. (2020) Healtheducation to prevent COVID-19 in schoolchildren: a call to action. Infectious diseases of poverty 9(1), 1-3.

Hancock T. S., Friedrichsen P. J., Kinslow A. T., Sadler T. D. (2019) Selecting socio-scientific issues for teaching. Science \& Education 28(6), 639-667. 
Herman B. C. (2015) The influence of global warming science views and sociocultural factors on willingness to mitigate global warming. Science Education 99(1), 1-38.

Isabella B. (2015) Graphical proof that vaccines work (with sources). https://cutt.ly/jv4dKnZ

Klosterman M. L., Sadler T. D. (2010) Multi - level assessment of scientific content knowledge gains associated with socioscientific issues - based instruction. International Journal of Science Education 32(8), 1017-1043.

Lachenbruch P. A. (2014) McNemar Test. Wiley StatsRef: Statistics Reference Online.

Lobera J., Cabrera P. (2021) Evaluación de la percepción social de aspectos científicos de la COVID-19. FECYT.

Lopes H., McKay V. (2020) Adult learning and education as a tool to contain pandemics: The COVID-19 experience. International review of education 66(4), 575-602.

Loomba S., de Figueiredo A., Piatek S., de Graaf K., Larson H. J. (2020) Measuring the Impact of Exposure to COVID-19 Vaccine Misinformation on Vaccine Intent in the UK and US. medRxiv.

Marton F. (1981) Phenomenography? Describing conceptions of the world around us. Instructional Science 10(2), $177-200$.

McIntyre L. (2019) The scientific attitude: Defending science from denial, fraud and pseudoscience. MIT Press.

Mellado Jiménez V., Borrachero A. B., Brígido M., Melo L. V., Dávila M. A., Conde M. C., ... Bermejo M. L. (2014) Las emociones en la enseñanza de las ciencias. Enseñanza de las Ciencias 32(3), 0011-36.

Morawska L., Tang J. W., Bahnfleth W., Bluyssen P. M., Boerstra A., Buonanno G., Haworth, C. (2020) How can airborne transmission of COVID-19 indoors be minimised?. Environment international 142, 105832.

Neupane B. B., Mainali S., Sharma A., Giri B. (2019) Optical microscopic study of surface morphology and filtering efficiency of face masks. PeerJ 7, e7142.

Okunlola M. A., Lamptey E., Senkyire E. K., Dorcas S., Dooshima B. A. (2020) Perceived Myths and Misconceptions about the Novel COVID-19 Outbreak. SciMedicine Journal 2(3), 108-11.

Page B. (2021) Attitudes to COVIS-19 vaccines. Ipsos MORI. https://cutt.ly/Vv4fnVx

Polack F. P., Thomas S. J., Kitchin N., Absalon J., Gurtman A., Lockhart S., ... Gruber W. C. (2020) Safety and efficacy of the BNT162b2 mRNA Covid-19 vaccine. New England Journal of Medicine 383(27), 2603-2615.

Puspitasari I. M., Yusuf L., Sinuraya R. K., Abdulah R., Koyama H. (2020) Knowledge, attitude, and practice during the COVID-19 pandemic: a review. Journal of Multidisciplinary Healthcare 13, 727-733.

Reiss M. J. (2020) Science Education in the Light of COVID-19. Science y Education 29(4), 1079-1092.

Romine W. L., Sadler T. D., Kinslow A. T. (2017) Assessment of scientific literacy: Development and validation of the Quantitative Assessment of Socio - Scientific Reasoning (QuASSR). Journal of Research in Science Teaching 54(2), 274-295.

Roozenbeek J., Schneider C. R., Dryhurst S., Kerr J., Freeman A. L., Recchia G., ... Van Der Linden S. (2020) Susceptibility to misinformation about COVID-19 around the world. Royal Society open science 7(10), 201199.

Sadler T. D. (2009) Situated learning in science education: socio - scientific issues as contexts for practice. Studies in science Education 45(1), 1-42.

Sadler T. D., Chambers F. W., Zeidler D. L. (2004) Student conceptualizations of the nature of science in response to a socioscientific issue. International Journal of Science Education 26(4), 387-409.

Salter M. (2021) 15 Examples of Diffusion in Real Life. Your Dictionari. Recuperado 19 de julio de 2021, de https:// examples.yourdictionary.com/examples-of-diffusion.html

Sinatra G. M., Kienhues D., Hofer B. K. (2014) Addressing challenges to public understanding of science: Epistemic cognition, motivated reasoning, and conceptual change. Educational Psychologist 49(2), 123-138.

Soares P., Rocha J. V., Moniz M., Gama A., Laires P. A., Pedro A. R., ... Nunes C. (2021) Factors Associated with COVID-19 Vaccine Hesitancy. Vaccines 9(3), 300.

Solbes J., Torres N. (2012) Análisis de las competencias de pensamiento crítico desde el aborde de las cuestiones sociocientíficas: un estudio en el ámbito universitario. Didáctica de las ciencias experimentales y sociales (26). 
Stolz M., Witteck T., Marks R., Eilks I. (2013) Reflecting socio-scientific issues for science education coming from the case of curriculum development on doping in chemistry education. Eurasia Journal of Mathematics, Science and Technology Education 9(4), 361-370.

Tang J., Bahnfleth W., Bluyssen P., Buonanno G., Jimenez J., Kurnitski J., Li Y., Miller S., Sekhar C., Morawska L., Marr L., Melikov A., Nazaroff W., Nielsen P., Tellier R., Wargocki P., Dancer S. (2021) Dismantling myths on the airborne transmission of severe acute respiratory syndrome coronavirus-2 (SARS-CoV-2). Journal of Hospital Infection 110, 89-96.

Treagust D. F., Chandrasegaran A. L., Crowley J., Yung B. H. W., Cheong I. P. A., Othman J. (2009) Evaluating students' understanding of kinetic particle theory concepts relating to the states of matter, changes of state and diffusion: a cross-national study. International Journal of Science and Mathematics Education 8(1), 141-164.

van Prooijen J. W. (2017) Why education predicts decreased belief in conspiracy theories. Applied cognitive psychology $31(1), 50-58$.

Verma S., Dhanak M., Frankenfield J. (2020) Visualizing the effectiveness of face masks in obstructing respiratory jets. Physics of Fluids 32(6), 061708.

Zeidler D. L. (2014) Socioscientific issues as a curriculum emphasis. Theory, research, and practice. In NG Lederman $y$ SK Abell (Eds.), Handbook of research on science education 2, 697-726.

Zeidler D. L., Herman B. C., Ruzek M., Linder A., Lin S. S. (2013) Cross - cultural epistemological orientations to socioscientific issues. Journal of Research in Science Teaching 50(3), 251-283.

Zeidler D. L., Walker K. A., Ackett W. A., Simmons M. L. (2002) Tangled up in views: Beliefs in the nature of science and responses to socioscientific dilemmas. Science education 86(3), 343-367.

\section{INFORMACIÓN ADICIONAL}

Para citar este artículo:: Portillo-Blanco, A., Ramón Díez, J., Barrutia, O., Garmendia, M. y Guisasola, J. (2022) Diseño y evaluación de una intervención educativa sobre la pandemia de la COVID-19 y las medidas de prevención. Revista Eureka sobre Enseñanza y Divulgación de las Ciencias 19(1), 1302. doi: 10.25267/ Rev_Eureka_ensen_divulg_cienc.2022.v19.i1.1302 